\title{
AN APPLICATION OF THE FINITE ELEMENT TECHNIQUE TO BOUNDARY VALUE PROBLEMS OF POTENTIAL FLOW
}

\author{
L. J. DOCTORS \\ Teaching Fellow and Research Assistant, \\ Department of Naval Architecture and Marine Engineering, \\ University of Michigan, Ann Arbor, Michigan
}

\section{SUMMARY}

This report summarizes an experimental application of the finite element approach to two-dimensional inviscid fluid flow.

The method results in a matrix equation relating the vector of velocities at the nodal points with the vector of singularities. The singularities, which are concentrated at the nodes, consist of a source and a vortex.

On solution of this equation (defining the flow in the region), boundary conditions must be stipulated. This usually involves setting the internal singularities to zero and making certain modifications to those lying on the boundary of the region.

Some results of the application to a particular problem are included in the paper.

\section{INTRODUCTION}

\section{History}

The finite element technique has been considerably developed in the last few years. This development was almost exclusively in the direction of stress analysis. ${ }^{1.2}$ The technique has, however, also been applied to other problems in continuum mechanics, for example, potential flow, by Zienkiewicz and others [References 1 (Chapter 10), 3 and 4].

This latter application is based on the 'extremum' method, also described by Berg. ${ }^{5}$ Here, a functional, representing an integrated quantity (the energy) over the region of interest is defined. It can be shown that the minimizing of this functional is equivalent to solving the original differential equation (Poisson or Laplace), describing the flow. This procedure leads to a set of linear equations which relate the potential function $\phi$ to the production of heat or matter $m$. One can then calculate the velocity distribution from the potential field.

The present work was motivated by an eventual hope to apply the technique to viscous flow, since there already exists a number of classical methods for solving potential flow problems.

\section{Present work}

In this paper, a different technique from that mentioned above will be dealt with. It is a slightly more physical approach. The method will be worked for a two-dimensional flow using the triangular element [Reference 1 (Chapter 3)].

The region is therefore subdivided into triangular elements as shown in Figure 1. In the corresponding stress analysis problem one normally deals with (a) the displacements at the nodes and (b) the associated forces. Here, we choose as our variables, (a) the velocities in the $x$ and $y$ 


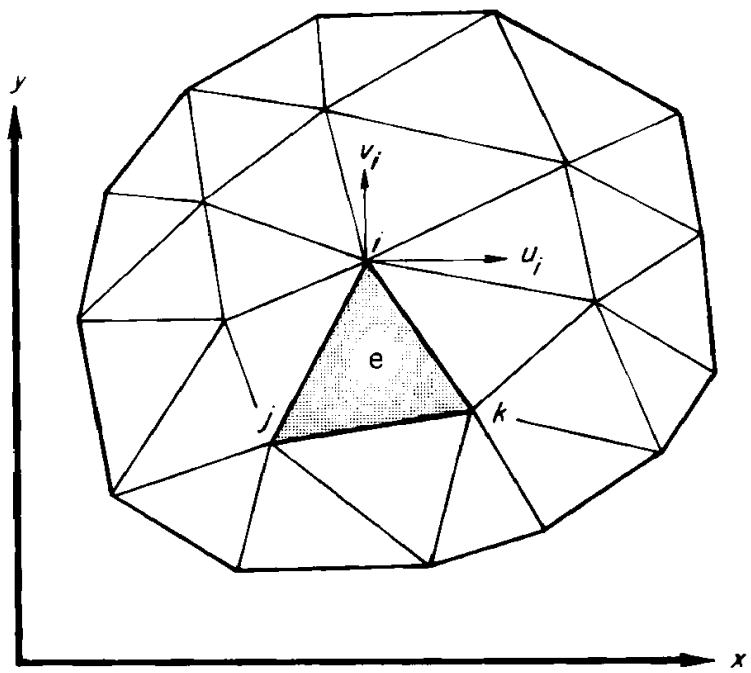

Figure 1. Subdivision of region into triangular elements

directions, $u$ and $v$ respectively, and (b) the volume source and circulation per unit thickness of the flow, $m$ and $\Gamma$, respectively.

As in other finite element approaches, the singularities ( $m$ and $\Gamma$ ) which may be distributed throughout the region have to be considered lumped at the nodes. For each element, then, we may define the vectors:

$$
\mathbf{f}=\left\{\begin{array}{c}
\mathbf{f}_{i}^{e} \\
\mathbf{f}_{j}^{e} \\
\mathbf{f}_{k}^{e}
\end{array}\right\} \text { with } \quad \mathbf{f}_{i}^{e}=\left\{\begin{array}{c}
m_{i}^{e} \\
\Gamma_{i}^{e}
\end{array}\right\}, \quad \text { etc. }
$$

and

$$
\mathbf{u}^{e}=\left\{\begin{array}{c}
\mathbf{u}_{i} \\
\mathbf{u}_{j} \\
\mathbf{u}_{k}
\end{array}\right\} \text { with } \mathbf{u}_{i}=\left\{\begin{array}{c}
u_{i} \\
v_{i}
\end{array}\right\}, \text { etc. }
$$

The velocity field in the whole region is defined by

and the singularity field is given by

$$
\mathbf{u}^{D}=\left\{\begin{array}{c}
\mathbf{u}_{1} \\
\mathbf{u}_{2} \\
\vdots \\
\mathbf{u}_{n}
\end{array}\right\}
$$

$$
\mathbf{f}^{D}=\left\{\begin{array}{c}
\mathbf{f}_{1} \\
\mathbf{f}_{2} \\
\vdots \\
\mathbf{f}_{n}
\end{array}\right\}
$$

where the total singularity $\mathbf{f}_{i}$ at node $i$ is obtained by adding the contributions $\mathbf{f}_{i}^{e}$, from the elements contiguous to that node.

In the following we will find a relationship between $\mathbf{u}^{D}$ and $\mathbf{f}^{D}$. 


\section{PROCEDURE}

\section{Element characteristics}

The first assumption to be made concerns the velocity distribution inside the element. The simplest is a linear one:

$$
\mathbf{u}=\lambda_{i} \mathbf{u}_{i}+\lambda_{j} \mathbf{u}_{j}+\lambda_{k} \mathbf{u}_{k}
$$

where $\lambda_{i}$, etc. are the so-called 'natural co-ordinates' and are given by

$$
\lambda_{i}=A_{i} / \Delta \text {, etc. }
$$

where $\Delta$ is the area of the element and $A_{i}$ is the area of the triangle subtended by the side opposite node $i$, as in Figure 2 . Note that this assumption for $\mathbf{u}$ will violate the Laplace equation within the element. Such a simplification is in the spirit of the finite element method and is tolerated in favour of satisfaction on the region as a whole, after the 'assembly' procedure.

$$
\begin{aligned}
x & =\sum \lambda_{j} x_{i} \\
\text { with } \lambda_{i} & =A_{i} / \Delta \\
2 \lambda_{i} & =a_{j}+b_{j} x+c_{j} y \\
a_{i} & =x_{j} y_{k}-x_{k} y_{j} \\
b_{i} & =y_{j}-y_{k} \\
c_{j} & =x_{k}-x_{j} \text { etc, cyclically }
\end{aligned}
$$

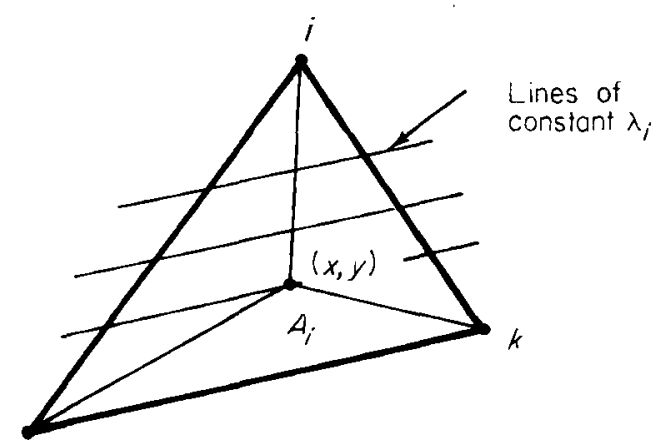

Figure 2. Illustration of the 'natural co-ordinate system'

Secondly, we must define a path of integration for the calculation of the singularities in terms of the velocities. This is shown in Figure 3. The path, as it cuts through the element, consists of the straight line:

$$
\lambda_{i}=\frac{1}{2}, \quad \lambda_{j}=\frac{1}{2}-\lambda_{k}, \quad 0 \leqslant \lambda_{k} \leqslant \frac{1}{2}
$$

The vector representing this line of integration within the element is given by

$$
\mathbf{s}=\lambda_{k}\left\{\begin{array}{c}
x_{k}-x_{j} \\
y_{k}-y_{j}
\end{array}\right\}
$$

and there are similar expressions for the vector path of integration for the singularities at nodes $j$ and $k$. Equation (8) can be further written as

where

$$
\mathbf{s}^{T}=\lambda_{k} \mathbf{x}^{e T} \mathbf{T}_{2}
$$

$$
\mathbf{x}^{e T^{\prime}}=\left(\mathbf{x}_{i}^{T}, \mathbf{x}_{j}^{T}, \mathbf{x}_{k}^{T}\right) \quad \text { with } \quad \mathbf{x}_{i}=\left\{\begin{array}{c}
x_{i} \\
y_{i}
\end{array}\right\} \text {, etc. }
$$




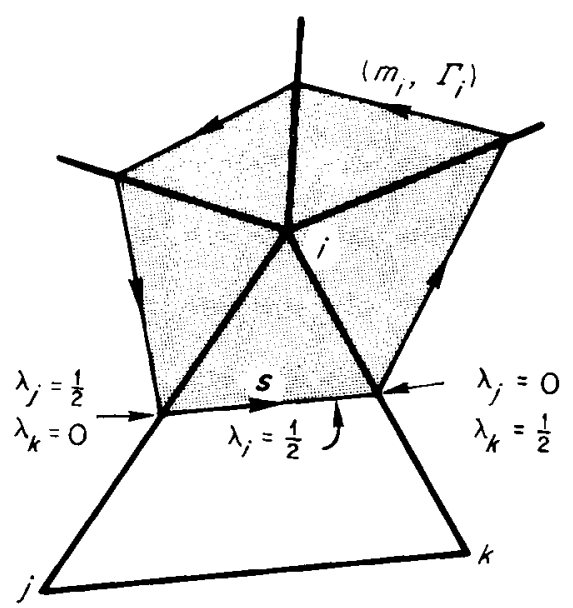

Figure 3. Path of integration connecting the midpoints of the sides of the triangle, for the calculation of $m$ and $\mathrm{I}$ and

$$
\mathbf{T}_{2}=\left[\begin{array}{c}
\mathbf{O} \\
-\mathbf{I} \\
\mathbf{I}
\end{array}\right]
$$

where $\mathbf{O}$ and $\mathbf{I}$ are respectively the null and identity matrices. The superscript $T$ indicates the transpose of a vector or matrix.

\section{Analysis}

The element of volume flux through the path of integration is given by

$$
\begin{aligned}
\mathrm{d} m_{i}^{e} & =\mathrm{d} \mathbf{s}^{T}\left[\begin{array}{cc}
0 & -1 \\
1 & 0
\end{array}\right] \mathbf{u} \\
& =\mathrm{d} \mathbf{s}^{T} \mathbf{T} \mathbf{u}
\end{aligned}
$$

T being a transformation matrix.

Integrating equation (12) along the path given by equation (7) we obtain for the flux through the path, from node $i$ within element $e$,

$$
m_{i}^{e}=\int_{\lambda_{k}=0}^{\frac{1}{2}} \mathrm{ds}^{T} \mathbf{T u}
$$

Substituting for $\mathbf{s}^{T}$ and $\mathbf{u}$ from equations (9) and (5), we obtain

or

$$
m_{i}^{e}=\mathbf{x}^{e T} \mathbf{T}_{2} \mathbf{T} \int_{\lambda_{k}=0}^{\frac{1}{2}}\left[\frac{1}{2} \mathbf{u}_{i}+\left(\frac{1}{2}-\lambda_{k}\right) \mathbf{u}_{j}+\lambda_{k} \mathbf{u}_{k}\right] \mathrm{d} \lambda_{k}
$$

Or, finally,

$$
m_{i}^{e}=\frac{1}{8} \mathbf{x}^{e T} \mathbf{T}_{2} \mathbf{T}\left(2 \mathbf{u}_{i}+\mathbf{u}_{j}+\mathbf{u}_{k}\right)
$$

where

$$
m_{i}^{e}=\mathbf{x}^{e T} \mathbf{R} \mathbf{u}^{e}
$$

$$
\mathbf{R}=\frac{1}{8}\left[\begin{array}{ccc}
\mathbf{O} & \mathbf{O} & \mathbf{O} \\
-2 \mathbf{T} & -\mathbf{T} & -\mathbf{T} \\
2 \mathbf{T} & \mathbf{T} & \mathbf{T}
\end{array}\right]
$$


Now the contributions to the flux of all the elements sharing the common node may be added:

$$
m_{i}=\sum_{\substack{e \\ \text { (adjoining } i \text { ) }}} \mathbf{x}^{e T} \mathbf{R} \mathbf{u}^{e}
$$

The element of circulation along the chosen path of integration is

$$
\mathrm{d} \Gamma_{i}^{e}=\mathrm{ds}{ }^{T} \mathbf{u}
$$

In a manner similar to that above we obtain for the entire circulation at nodal point $i$,

where

$$
\Gamma_{i}=\sum_{\substack{e \\ \text { (adjoining } i)}} x^{e T} \mathrm{Su}^{e}
$$

$$
\mathbf{S}=\frac{1}{8}\left[\begin{array}{ccc}
\mathbf{O} & \mathbf{0} & \mathbf{0} \\
-2 \mathbf{I} & -\mathbf{I} & -\mathbf{I} \\
2 \mathbf{I} & \mathbf{I} & \mathbf{I}
\end{array}\right]
$$

The 'assembly' of the elements can now follow. It will be seen that equations (16) and (18), for the flux and circulation, can be expressed as a linear combination of the velocities. Thus, one can assemble the expressions (16) and (18) for each of the $n$ nodes into a system of $2 n$ linear equations expressible in matrix form:

$$
\mathbf{K}^{D} \mathbf{u}^{D}=\mathbf{f}^{D}
$$

Here, the control matrix $\mathbf{K}^{D}$ corresponds to the stiffness matrix used in problems of stress analysis.

\section{Discussion of control matrix}

The matrix $\mathbf{K}^{D}$ has the following three properties to be noted.

Similar to stiffness matrices it is singular and, in this case, to two degrees. One degree of singularity represents the fact that the fluxes $m_{i}$ must sum to zero for continuity to be satisfied on the region as a whole. The second degree of singularity is similarly due to the vortices $\Gamma_{i}$ summing to zero. Thus the even and odd rows are separately singular to one degree.

Secondly, it is band limited. The semi-bandwidth is given by

$$
b=2(\max |i-j|+1)
$$

where the maximum operation is taken over all pairs of $i, j$ (both being in the same element). This fact can be used to reduce the amount of work to invert the matrix.

Thirdly, contrary to stiffness matrices, $\mathbf{K}^{D}$ is not symmetric.

\section{Boundary conditions}

Generally speaking, if one is dealing with a potential flow, the singularities $\mathbf{f}_{i}$ will be put to zero in equation (20). This will be true for all internal nodes, but not for those on the boundary.

This is because the integration loops, shown in Figure 3, will not be closed for boundary nodes. If, for example, node $i$ is on a boundary represented by a solid wall (non-conducting boundary) then there is a geometric requirement that the flow be tangential to it. Hence, the circulation $\Gamma_{i}$ will be given by the product of the length of the integration loop in contact with the boundary, and the mean tangential velocity (which is usually unknown). However, the flux $m_{i}$ would still be zero.

The difficulty can be resolved by completing the loop for one or other of the components of singularity, as shown in Figure 4(a). The modified singularity at node $i$ is then defined on this completed loop. This is readily achieved on the computer by assuming a fictitious node $i^{\prime}$ near $i$, Figure 4(b), thus causing $i$ to become 'internal'. Choosing the co-ordinates of $i$ ' to be those of $i$ causes no problems. With the notation that $m^{*}$ and $\Gamma^{*}$ represent such modified singularities, we can now state the boundary conditions for the following cases. 

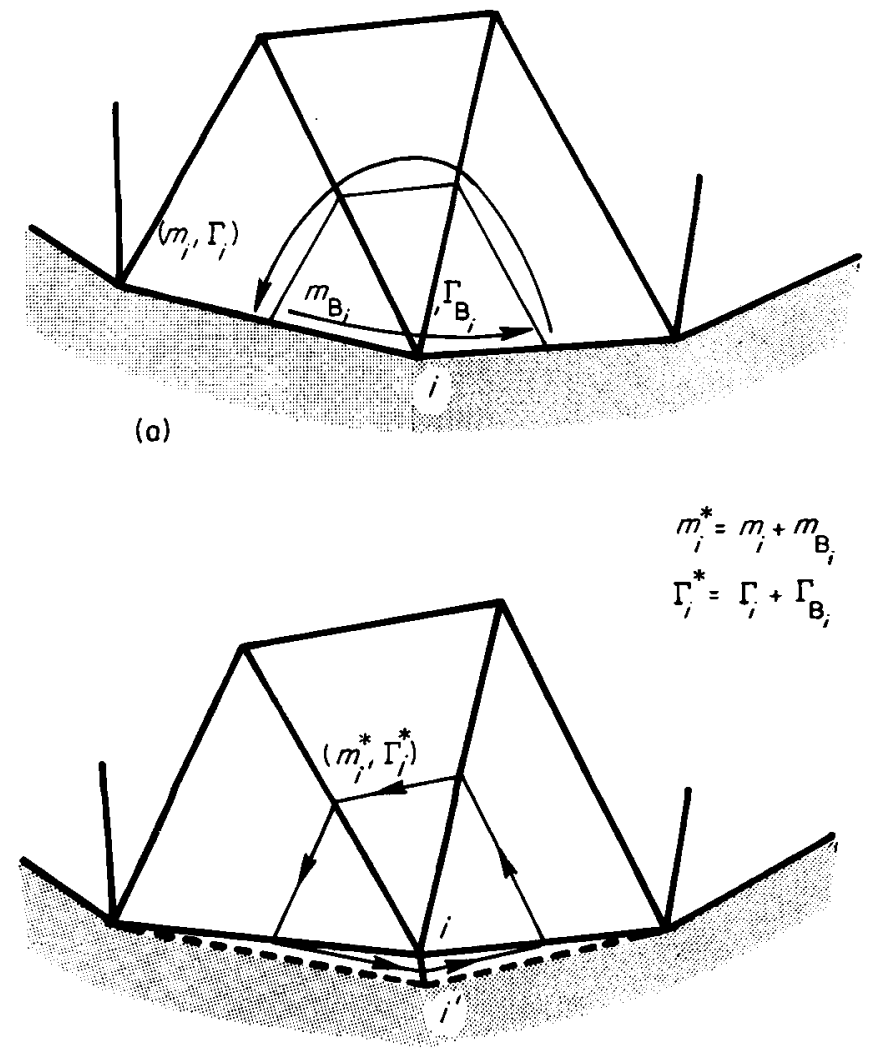

(b)

Figure 4. (a) Boundary modifications for node $i$. (b) Showing use of fictitious node $i^{\prime}$

1. Non-conducting boundary (flow tangential):

$$
m_{i}=0, \quad \Gamma_{i}^{*}=0
$$

2. Purely conducting boundary (flow normal):

$$
m_{i}^{*}=0, \quad \Gamma_{i}=0
$$

3. Velocity-specified boundary:

$$
\begin{aligned}
& u_{i} \text { and } v_{i} \text { given on the boundary, and the appropriate rows of } \\
& \mathbf{K}^{D} \text { excluded from the inverting process }
\end{aligned}
$$

4. Singularity-specified boundary:

$$
m_{i} \text { and } \Gamma_{i} \text { given on the boundary }
$$

\section{A TEST PROBLEM}

One of the problems for which the method was tested is shown in Figure 5. The flow is parallel to the solid boundaries and normal to the open ones, and therefore corresponds to part of the infinite flow around a circular cylinder. The three following techniques for obtaining a solution were tried: 


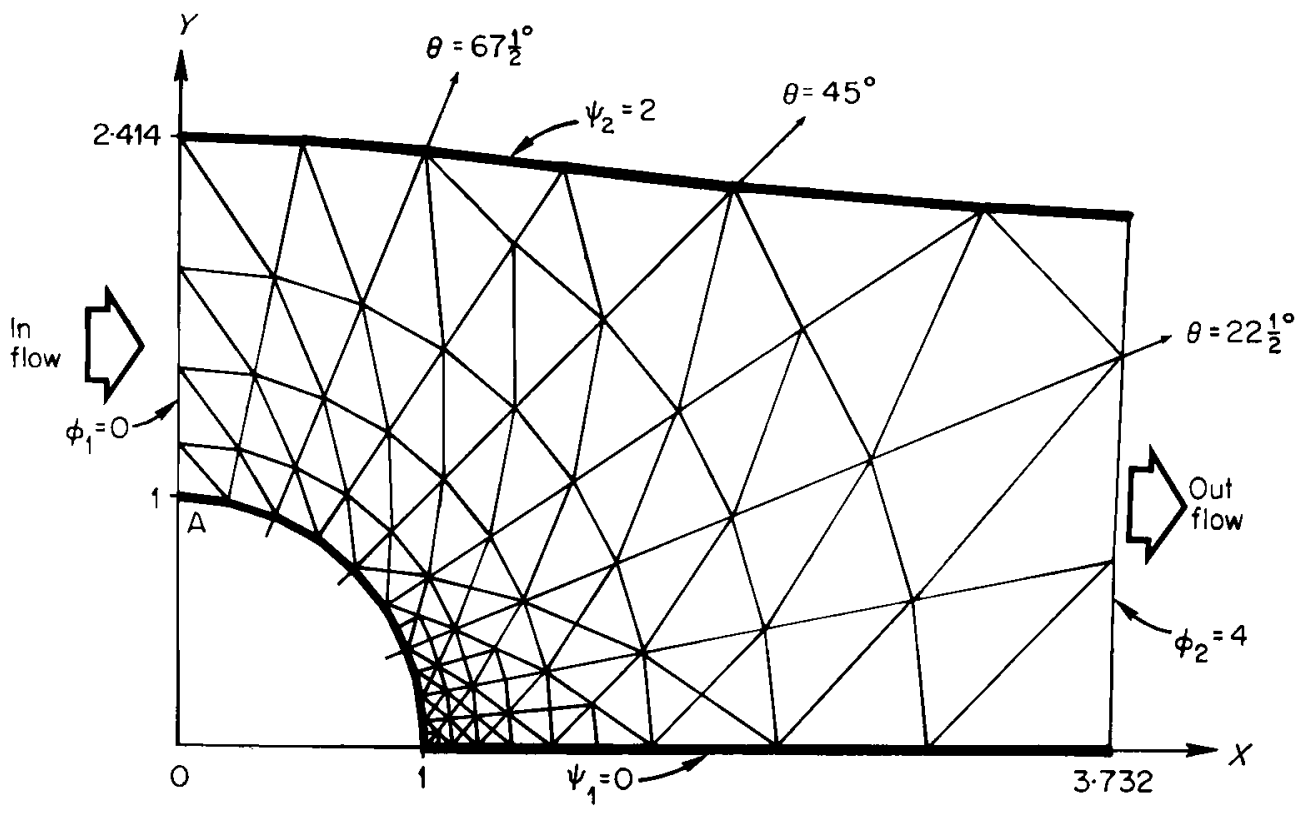

Figure 5. Test problem described in the text

\section{Modified boundaries}

Here conditions (22) and (23) were applied to the appropriate parts of the boundary. Normal Gaussian elimination procedure was used, and the problem was made non-singular by stating the velocity at node $\mathrm{A}$ in Figure 5. The results are compared with the analytic solution in Figure 6.

\section{Velocity-specified boundary}

The boundary velocities were obtained from the analytic solution and used in condition (24). These results are also shown in Figure 6.

\section{Singularity-specified boundary}

In this case, singularities calculated from differences in the complex potential of the exact solution were lumped at the nodes according to condition (25). The accuracy of the results was so poor that there was no visible relationship between the finite element solution and the exact one.

\section{CONCLUDING REMARKS}

\section{Discussion of results}

The present approach to the solution of potential flow gives useful results. The methods of applying the first two boundary conditions described were tried in other situations, for example the flow about a concentrated source and that about a concentrated vortex. In these two cases the solutions exhibited the correct velocity distributions to a similar accuracy.

The poor results of the singularity-specified boundary, condition (25) were traced to the matrix $\mathbf{K}^{D}$ being ill-conditioned. Minor changes in the specified singularities on the boundaries could result in vastly different solutions, indicating the unreliability of this method.

\section{Further work}

The results obtained so far indicate the experimental nature of this method. Questions still to be answered concern the matrix $\mathbf{K}^{D}$. Why is it ill-conditioned when the singularities on the boundary are specified? What is the best technique for carrying out the inversion process? 
L. J. DOCTORS
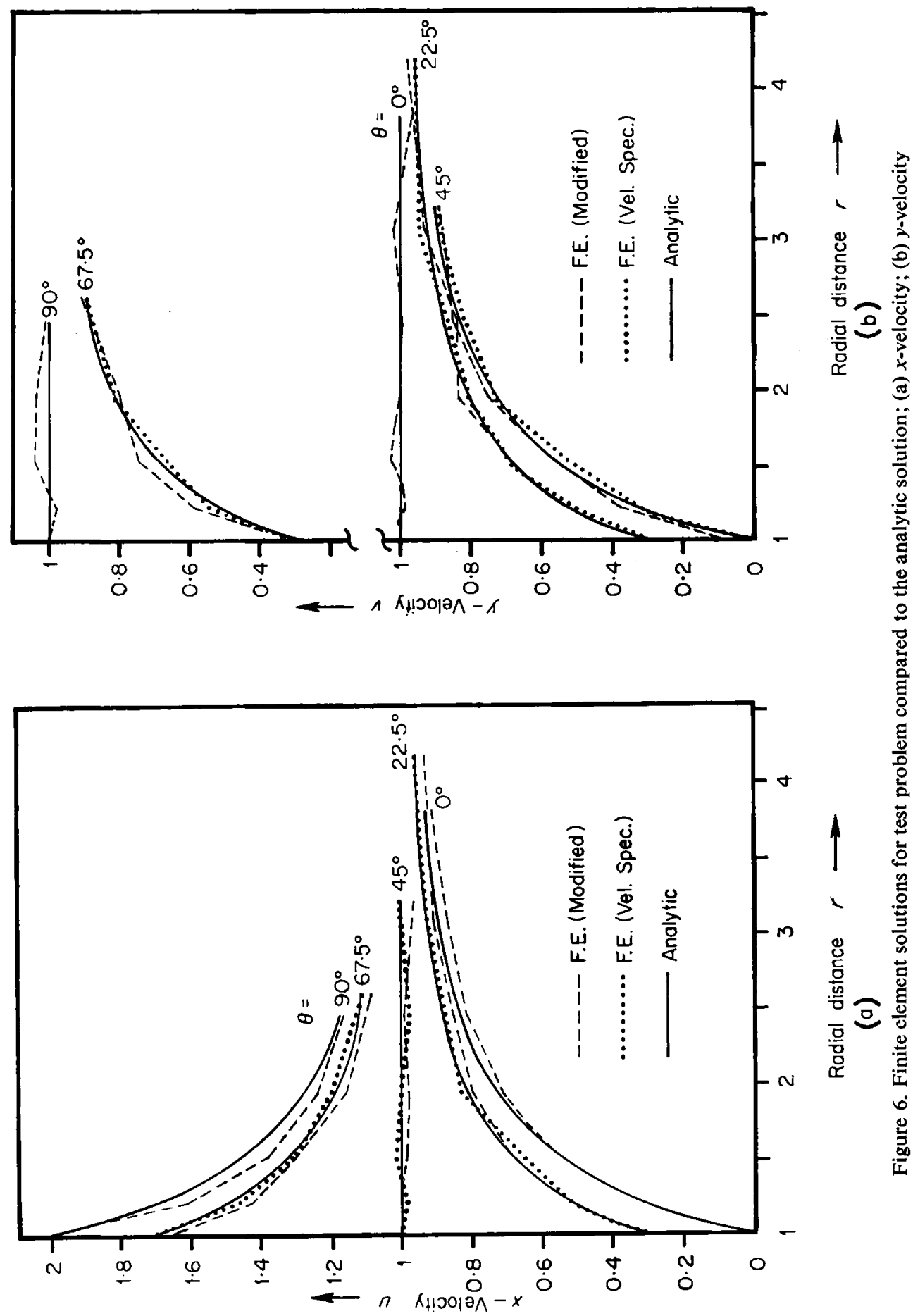
These questions being answered it would be worth while to investigate the possibility of extending the analysis to viscous flow. In this case, at the internal nodes, $m_{i}$ will still be zero but $\Gamma_{i}$ would be non-zero. However, it is not certain at this stage whether a non-linear problem (the case of viscous flow) could be handled directly by such a matrix method, although the finite element aspect should not cause any trouble.

\section{ACKNOWLEDGEMENTS}

The writer gratefully acknowledges the assistance given him by Professor J. Moe, Department of Ship Structures, Technical University of Norway, Trondheim, who first suggested this project, and also by Professor $H$. Nowacki of the Department of Naval Architecture and Marine Engineering, University of Michigan, Ann Arbor, Michigan, for his help and advice.

\section{APPENDIX}

\section{Notation}

$A=$ Area of subtriangle, see Figure 2

$b=$ Semi-bandwidth of matrix $\mathbf{K}$

$D=$ Superscript referring to total region of flow

$e=$ Superscript referring to an individual element

$\mathbf{f}=$ Singularity vector

$i, j, k=$ Subscripts referring to nodal points

$I=$ Identity matrix of dimension 2

$\mathbf{K}=$ Control matrix relating $\mathbf{f}$ and $\mathbf{u}$

$m=$ Flux strength

$n=$ Number of nodes in the region

$\mathbf{O}=$ Null or zero matrix of dimension 2

$\mathbf{R}=$ Matrix defined by equation (15)

$r=$ Radial distance in Figure $5=\sqrt{(}\left(x^{2}+y^{2}\right)$

$\mathbf{s}=$ Vector position on integration path, see equation (8)

$\mathbf{S}=$ Matrix defined by equation (19)

$\mathbf{T}=\left[\begin{array}{cc}0 & -1 \\ 1 & 0\end{array}\right]$

$\mathrm{T}_{2}=(\mathbf{O},-\mathbf{I}, \mathbf{I})^{T^{\prime}}$

$T=$ Indicates the transpose of a vector or matrix

$u, v=x$ and $y$ components of $\mathbf{u}$

$\mathbf{u}=$ Velocity vector at a point

$x, y=$ Cartesian co-ordinates, components of $\mathbf{x}$

$\mathbf{x}=$ Vector position of a point

$\Gamma=$ Vortex strength

$\Delta=$ Area of triangular element

$\theta=$ Angle of radius vector in Figure $5=\tan ^{-1}(y / x)$

$\lambda=$ 'Natural' co-ordinate, see Figure 2

$\phi=$ Velocity potential

$\psi=$ Stream function

* = Indicates modified singularities, see Figure 4 
L. J. DOCTORS

\section{REFERENCES}

1. O. C. Zienkiewicz, The Finite Element Method in Structural and Continuum Mechanics, McGraw-Hill, New York, 1967.

2. D. C. Tolefson and L. Brand, 'Introduction to finite element methods of structural analysis', Mar. Technol., 5, 4, 331-346 (1968).

3. O. C. Zienkiewicz and Y. K. Cheung, 'Finite elements in the solution of field problems', Engineer, London, 220, 5722, 507-510 (1965).

4. O. C. Zienkiewicz, P. Mayer and Y. K. Cheung, 'Solution of anisotropic seepage by finite elements', J. Eng. Mech. Div., Proc. Am. Soc. civ. Eng., 92, EM 1, 111 (1966).

5. P. N. Berg, 'Calculus of variation', in Handbook of Engineering Mechanics (Ed. W. Flügge), McGraw Hill, New York, 1962, Chap. 16. 